\title{
Les écoles privées en Scandinavie
}

Réglementation publique et fragmentation

Private schools in Scandinavia. Public regulation and fragmentation

Las escuelas privadas en Escandinavia. Reglamentación pública y fragmentación

\section{Signe Bock Segaard}

Traducteur : Eva Loechner

\section{CpenEdition}

\section{Journals}

Édition électronique

URL : https://journals.openedition.org/ries/6073

DOI : 10.4000/ries.6073

ISSN : 2261-4265

Éditeur

France Education international

Édition imprimée

Date de publication : 1 décembre 2017

Pagination : 103-114

ISBN : 978-2-85420-616-6

ISSN : 1254-4590

Référence électronique

Signe Bock Segaard, "Les écoles privées en Scandinavie », Revue internationale d'éducation de Sèvres

[En ligne], 76 | décembre 2017, mis en ligne le 01 décembre 2019, consulté le 25 juin 2021. URL http://journals.openedition.org/ries/6073 ; DOI : https://doi.org/10.4000/ries.6073 


\title{
Les écoles privées en Scandinavie
}

Réglementation publique et fragmentation*

\author{
Signe Bock Segaard \\ Institute for Social Research, Oslo
}

Cet article s'intéresse à la question de la fragmentation au sein des systèmes scolaires du point de vue des politiques publiques, et tout particulièrement des régulations publiques qui affectent les écoles primaires et les établissements du secondaire inférieur (à but lucratif ou non) dans trois pays scandinaves, la Norvège, le Danemark et la Suède. Ces régulations portent sur des points qui peuvent être d'importantes sources de fragmentation, tant du côté des écoles que de leurs usagers. Du côté des écoles, la fragmentation peut naître des différents types d'établissements privés autorisés par l'État, ainsi que de la latitude qui leur est offerte en termes d'objectifs, de résultats, de pratiques et de formes scolaires. Pour le dire autrement, la régulation publique du secteur éducatif privé est susceptible d'affecter directement le degré de fragmentation et la diversité du système éducatif du pays concerné. Au niveau individuel, les réglementations portant sur les droits et les devoirs des usagers (parents et élèves) sont également susceptibles d'entraîner une certaine fragmentation du système scolaire. Au cours des dernières décennies, la promotion d'une citoyenneté active, incluant la liberté de choix en matière d'éducation, a été et continue d'être un objectif prioritaire dans ces trois pays scandinaves, comme le préconise l'Unesco avec "l'éducation à la citoyenneté »(Unesco, 2010). Cet objectif implique que les élèves soient considérés comme des individus dotés de droits et de devoirs, pleinement acteurs de la vie démocratique.

Nous soutenons notamment dans cet article que les politiques publiques et leurs changements peuvent être compris sous l'angle des valeurs. Dans le cas des systèmes éducatifs scandinaves, on peut considérer que les caractéristiques des régulations publiques sont le reflet de valeurs contradictoires jusqu'à un certain point : l'égalitarisme et l'universalisme en matière d'éducation se heurtent à la fragmentation, à la diversité et à l'individualisme. Ce conflit de valeurs est omniprésent dans le débat public sur l'école et les politiques éducatives dans les pays scandinaves. L'une des explications tient au fait qu'historiquement, tous les systèmes éducatifs scandinaves reflètent l'accent mis par le modèle socialdémocrate de l'État providence sur le droit universel à l'éducation et sur une "école pour tous » unifiée, caractérisée par des investissements publics

* Article traduit par Eva Loechner. 
importants, un secteur public de l'éducation très développé, et une régulation englobant tant le secteur public qu'un secteur privé restreint (Wiborg 2013). Cette régulation, guidée par des valeurs égalitaristes et inclusives, a garanti pendant plus d'un siècle l'existence d'un système scolaire relativement unifié au sein des pays scandinaves (Balle, 2012). Toutefois, depuis quelques décennies, cette quasi-absence de fragmentation commence à être remise en cause, sous l'effet de politiques publiques d'éducation d'inspiration néo-libérale (voir par exemple Vockmar et Wiborg, 2014). Ces changements de politique ont eu lieu dans les trois pays scandinaves, mais de façon particulièrement significative en Suède, où les établissements privés, et notamment ceux à but lucratif, se sont vu octroyer un rôle plus important et de meilleures conditions de fonctionnement au cours de ces réformes fondées sur les mécanismes du marché. Avant le milieu des années 1990, le système scolaire suédois ne comportait quasiment que des écoles publiques. Dans l'éducation primaire et secondaire inférieure, ces changements se manifestent aujourd'hui par la coexistence d'établissements publics avec des établissements privés à but lucratif. Tandis que le nombre d'établissements privés à but non lucratif est relativement important au Danemark, les établissements à but lucratif ont un rôle plus significatif en Suède. En Norvège, la part de ces deux types d'acteurs privés est, en réalité, assez modeste. Il faut toutefois rappeler que la place du secteur privé - qu'il soit à but lucratif ou non - demeure relativement faible dans les pays scandinaves, en comparaison de ce qui se passe à l'échelle internationale.

Cet article vise à se demander dans quelle mesure les régulations publiques du secteur privé éducatif conditionnent la fragmentation des systèmes scolaires scandinaves. Le point de départ est que le potentiel de fragmentation des systèmes scolaires scandinaves ne peut être compris que si l'on prend en compte les régulations publiques en général, et les conditions légales de fonctionnement des établissements privés en particulier. Cet article étudie ces questions en s'appuyant sur une analyse détaillée de textes réglementaires publics, tels que des textes de lois, des bulletins et directives officiels ${ }^{1}$.

Dans la section qui suit, nous nous intéressons aux régulations nationales au niveau des établissements, avant d'examiner la régulation des droits et devoirs des usagers. En conclusion, l'article revient sur les contextes nationaux et propose d'expliquer les différences en matière de régulation par des raisons sous-jacentes qui tiennent aux traditions historiques et politiques de chaque pays.

1. Les établissements privés sont soumis à la législation nationale : Friskoleloven au Danemark (LBK nr 917 af 13/08/2014), Skollag en Suède (SFS 2010:800), et Lov om frittståande skolar en Norvège (LOV-2003-07-04-84), outre divers textes réglementaires. Sur ces textes, voir Segaard (2015) et Saglie et Segaard (2017). Au Danemark, les pensionnats sont soumis à une autre législation que le Friskoleloven et ne sont pas pris en compte dans cet article. Il convient toutefois de mentionner que ce sont des établissements à but non lucratif. Ce genre d'établissements est rare en Norvège et en Suède. 


\section{LES TYPES D'ÉTABLISSEMENTS PRIVÉS ET LEUR LIBERTÉ DE FONCTIONNEMENT}

Traditionnellement, le secteur public tient le rôle le plus important dans le domaine scolaire en Norvège, au Danemark et en Suède. Un trait commun aux trois pays est que la grande majorité des élèves de primaire et de secondaire inférieur fréquentent une école municipale. Il existe en revanche quelques différences, s'agissant de la répartition des élèves entre le secteur public et le secteur privé. Selon les statistiques officielles pour l'année scolaire 2016-2017 (fournies par le ministère norvégien de l'éducation et de la formation, l'Office des statistiques suédois, le ministère danois de l'éducation), le pourcentage d'écoles primaires et d'établissements du secondaire inférieur privés est de $8,3 \%$ en Norvège, de $16,9 \%$ en Suède et de $22,9 \%$ au Danemark $(33,3 \%$ si l'on prend en compte les pensionnats). Comme ces écoles privées sont en moyenne plus petites que les écoles publiques, la part des élèves qui fréquentent un établissement privé est cependant moindre : $3,6 \%$ en Norvège, $14,6 \%$ en Suède et $16,7 \%$ au Danemark (20,6\% si l'on prend en compte les élèves scolarisés dans un pensionnat).

Le problème de ces statistiques officielles est qu'elles ne font pas de distinction entre les différents types d'institutions, comme par exemple entre les établissements à but lucratif et ceux à but non lucratif. Comme nous le verrons, cette distinction est cruciale lorsque l'on compare les politiques éducatives des trois pays. En Suède, les écoles primaires et établissements du secondaire inférieur privés qui reçoivent des financements publics ont le droit d'être gérés comme des entreprises commerciales. Ce n'est pas possible en Norvège et au Danemark. Environ 64 \% des élèves scolarisés dans le privé en Suède le sont dans un établissement à but lucratif (Sahlgren, 2016), ce qui signifie que ce type d'école attire près d'un élève sur dix. Les établissements privés en Suède sont totalement financés par le secteur public et ne sont pas autorisés à faire payer des frais de scolarité. Ce n'est pas le cas au Danemark et en Norvège, où les écoles privées ne sont qu'en partie financées par l'État. Les écoles privées danoises et norvégiennes sont donc autorisées à appliquer des frais de scolarité - et l'on considère que c'est normal. Dans ces deux pays, les financements doivent également obligatoirement être utilisés à des fins éducatives. En outre, au Danemark, la loi exige que les écoles privées soient gérées par des institutions à but non lucratif. Il est à l'évidence très avantageux d'être un opérateur à but lucratif au sein du système d'éducation primaire suédois, où les écoles sont entièrement financées par le secteur public: des profits peuvent être reversés aux propriétaires, et les autorités n'ont en pratique aucun regard sur les comptes de l'établissement (contrairement aux autorités danoises et norvégiennes). En d'autres termes, les propriétaires d'écoles à but lucratif en Suède opèrent dans un cadre économique beaucoup plus libre. Au vu de la liberté qui leur est 
accordée en termes de gestion comptable, les établissements privés suédois jouissent donc d'une position unique. Un effet indésirable peut être que ces écoles sont gérées dans un but mercantile de maximisation des profits aux dépens des critères éducatifs.

Dans les trois pays, les écoles primaires et les établissements du secondaire inférieur sont sous la juridiction des municipalités, qui ont l'obligation de scolariser tous les enfants de cette tranche d'âge. Toutefois, la scolarisation en établissement privé pour ces enfants est soumise à un système d'homologation au niveau national. Dans chaque pays, une institution publique est chargée d'homologuer les établissements privés (le ministère norvégien de l'éducation et de la formation, le ministère danois de l'enfance, de l'éducation et de l'égalité entre les sexes, l'inspection des établissements scolaires suédois). Pour le primaire et le collège, les acteurs non publics doivent donc demander l'autorisation de créer une école et un organisme public leur accorde ou non cette autorisation. Un tel système d'accréditation garantit le respect, de la part des établissements privés, de régulations nationales relatives à l'organisation et au contenu éducatif. Grâce à ce système, on peut considérer que, dans les trois pays, les autorités nationales exercent un contrôle indirect sur ces écoles, en définissant des critères et conditions à respecter pour obtenir l'accréditation. En outre, si ces critères ne sont pas respectés, cette homologation peut être retirée aux écoles. Les rapports entre les autorités et les établissements privés sont donc définis par un contrat, avec des critères d'homologation plus ou moins détaillés.

Toutefois, l'étendue de ces contrôles nationaux et le rôle de chaque acteur - autorités comme établissements - varient significativement d'un pays à l'autre. L'objet des régulations, tout comme la façon dont elles promeuvent et encouragent des phénomènes de fragmentation éducative basés sur des valeurs, diffèrent également. Les écoles privées danoises jouissent d'une plus grande liberté en termes de contenu éducatif et de valeurs. Un élément central de la politique scolaire danoise est que le contenu éducatif doit correspondre aux valeurs fondamentales de l'établissement et que les usagers potentiels doivent en être informés. Les réglementations stipulent d'ailleurs que les établissements ont le pouvoir de définir leurs propres contenus et méthodes éducatives en fonction de leurs valeurs fondamentales et de leurs caractéristiques distinctives. En d'autres termes, les autorités danoises ne contraignent pas les différents types d'établissements privés en termes de pédagogie, de valeurs, de croyances religieuses, de type de public, etc. En Norvège, une condition primordiale pour obtenir l'homologation est que l'école fonctionne sur la base de ce que la loi définit comme des "principes distincts ", à savoir : 1) une croyance religieuse ; 2) une méthode éducative alternative et différente de celle appliquée dans l'école publique mais reconnue par l'État (Steiner ou Montessori) ; 3) l'enseignement à l'étranger ; 4) l'enseignement destiné aux enfants en situation de handicap. En outre, en Norvège, et de façon encore plus prononcée en Suède, l'État définit également des standards détaillés pour l'éducation. Les écoles privées doivent se 
plier aux standards définis pour les écoles publiques, en conséquence de quoi la diversité des valeurs au sein du système éducatif est en grande partie régulée par des politiques publiques. En d'autres termes, les critères nationaux pour le contenu et l'organisation de l'enseignement nous apparaissent moins stricts au Danemark qu'en Norvège et en Suède. Alors que l'on peut considérer que les établissements du secteur public définissent un standard minimal pour les établissements du primaire et du secondaire inférieur au Danemark, les établissements du secteur public constituent plutôt, en Suède et en Norvège, un modèle à suivre pour les établissements privés. Ces régulations différentes ont probablement pour conséquence davantage d'uniformité et d'homogénéité entre le secteur public et le secteur privé en Norvège et en Suède, mais davantage de fragmentation et de diversité au Danemark.

Afin de garantir la diversité du système éducatif, les autorités danoises accordent également une latitude considérable aux écoles privées. Les écoles privées danoises peuvent rejeter des inscriptions d'élèves, même s'il leur reste de la place. Dans de telles conditions, une école est en mesure de cultiver sa différence d'image. L'ensemble du système scolaire primaire et secondaire inférieur gagne ainsi en diversité. À l'inverse, les écoles privées suédoises et norvégiennes ne peuvent en général pas refuser une candidature lorsqu'il leur reste de la place. Ceci peut être perçu comme une façon de garantir la liberté de choix des usagers. En pratique, le droit démocratique des usagers à choisir librement leur école est donc susceptible de nuire aux alternatives aux écoles publiques et, par conséquent, de réduire la diversité de l'offre visée en principe par les autorités. En effet, l'impossibilité pour les établissements de refuser des élèves limite leur liberté d'action.

\section{DROITS ET DEVOIRS DES USAGERS}

Les sources de fragmentation au sein des systèmes scolaires peuvent également être observées au niveau individuel, selon l'accent mis par les régulations publiques sur l'implication et les droits des usagers, et sur le développement d'une gamme plus large de services éducatifs, dans le but d'offrir une réelle liberté de choix entre diverses offres. Dans chacun des trois pays, l'une des priorités des politiques éducatives est de promouvoir la connaissance personnelle et le développement social des élèves, et de les préparer à devenir des citoyens actifs au sein d'une société démocratique ${ }^{2}$. Bien qu'il existe certaines différences, la liberté de choix des usagers est un droit fondamental au primaire et dans le secondaire inférieur en Norvège, en Suède et au Danemark. Le marché (c'est-àdire les usagers, à travers leurs choix individuels) contribue à façonner le système

2. Voir en Suède SFS 2010, au Danemark VEJ nº 145 af 23/07/2001, et en Norvège LOV-2003-07-04-84. 
éducatif. Toutefois, les conditions selon lesquelles les usagers peuvent faire un choix informé varient entre les trois pays, du moins en termes de régulation publique. La loi danoise, par exemple, oblige les écoles à informer les usagers - y compris les usagers potentiels - via leur site web. Le contenu de l'information et les canaux de diffusion sont clairement définis. De telles obligations sont largement absentes des régulations scolaires correspondantes en Suède et en Norvège.

Ce devoir statutaire d'information des usagers au Danemark peut être envisagé comme une condition fondamentale de l'exercice d'une citoyenneté active et éclairée par les usagers. Si ces derniers n'ont pas connaissance des différentes options qui s'offrent à eux et des conséquences qu'elles entraînent, proposer une offre scolaire diversifiée n'a guère de sens. Dans le pire des cas, on peut même considérer que l'inégalité de l'accès à l'information favorise l'inégalité des chances en matière d'éducation. Il est certain que chaque choix a des conséquences, en particulier quant aux droits et devoirs auxquels les parents acceptent de se conformer. Le Danemark se démarque également dans ce domaine. Les parents d'enfants scolarisés dans une école privée au Danemark ont un certain nombre d'obligations et doivent participer à la gestion de l'école. Un groupe de parents (appelé le "cercle des parents ») est formellement chargé de superviser l'école. Plus généralement, ce type de gouvernance collaborative est caractéristique du système danois de supervision des écoles privées. Non seulement les parents sont impliqués mais l'école elle-même peut choisir de jouer un rôle important en procédant à son auto-évaluation. Pour ce faire, un modèle d'auto-évaluation est mis à sa disposition par une association scolaire (c'est-à-dire une organisation nationale pour les écoles privées d'un certain type, comme les écoles confessionnelles, les écoles à destination de la minorité germanophone ou les écoles Waldorf-Steiner), après avoir été approuvé par les services nationaux d'inspection des écoles. L'inclusion des parents et d'organisations à but non lucratif dans la supervision des écoles est un exemple frappant de la façon dont la gouvernance collaborative entraîne des phénomènes d'auto-régulation au sein d'un cadre politique et institutionnel spécifique au niveau national (Sørensen et Torfing, 2008). Toutefois, il existe peu de collaboration directe entre les écoles à but non lucratif et les municipalités (Thøgersen, 2017). En d'autres termes, l'exercice d'un contrôle indirect à travers la gouvernance collaborative semble être limité à la relation entre l'établissement privé et l'État.

Ce système de supervision danois diffère sensiblement du modèle suédois, où de telles formes de gouvernance collaborative sont rares sinon inexistantes. Le système de supervision, en Suède, est centralisé et géré par l'État, avec très peu d'implication de la part des usagers ou des parents. De plus, la régulation suédoise prévoit que c'est au chef d'établissement de décider de l'organisation interne de son école. En conséquence, les textes ne disent pas grand-chose des devoirs et responsabilités des usagers (parents et enfants) en termes de 
participation citoyenne. Le peu que la loi mentionne au sujet de la participation et de l'implication des usagers renvoie davantage à leurs droits qu'à leurs devoirs. De plus, les régulations suédoises n'accordent aucun rôle aux usagers au sein des organes de direction des établissements. En Norvège, les usagers peuvent être impliqués, mais ne font l'objet d'aucun droit ou obligation formellement définis comme c'est le cas au Danemark.

Le tableau 1 résume les principaux points communs et différences entre les établissements du primaire et du secondaire inférieur au Danemark, en Suède et en Norvège.

Tableau 1. Caractéristiques de la régulation nationale des établissements privés dans les pays scandinaves

\begin{tabular}{|c|c|c|}
\hline Danemark & Suède & Norvège \\
\hline Organisation à but non lucratif & $\begin{array}{l}\text { Organisation à but commercial / } \\
\text { à but non lucratif }\end{array}$ & Organisation à but non lucratif \\
\hline Institution indépendante & Aucune régulation & Aucune régulation \\
\hline $\begin{array}{l}\text { Partiellement financée } \\
\text { par l'État }\end{array}$ & $\begin{array}{l}\text { Entièrement financée } \\
\text { par l'État }\end{array}$ & $\begin{array}{l}\text { Partiellement financée } \\
\text { par l'État }\end{array}$ \\
\hline Contrôle financier strict & Contrôle financier limité & Contrôle financier strict \\
\hline $\begin{array}{l}\text { Grande liberté } \\
\text { de fonctionnement } \\
\text { - Contenu éducatif } \\
\text { - Valeurs et normes } \\
\text { - Sélection des élèves }\end{array}$ & $\begin{array}{l}\text { Liberté de fonctionnement } \\
\text { plus limitée } \\
\text { - Contenu éducatif } \\
\text { - Valeurs et normes } \\
\text { - Absence de sélection } \\
\text { des élèves }\end{array}$ & $\begin{array}{l}\text { Liberté de fonctionnement } \\
\text { plus limitée } \\
\text { - Contenu éducatif } \\
\text { - Valeurs et normes } \\
\text { - Absence de sélection } \\
\text { des élèves }\end{array}$ \\
\hline $\begin{array}{l}\text { Gouvernance collaborative } \\
\text { très étendue } \\
\text { - Les parents et les } \\
\text { établissements eux-mêmes } \\
\text { ont des droits et des devoirs, } \\
\text { tels que l'obligation } \\
\text { de supervision } \\
\text { - Les associations nationales } \\
\text { d'établissements privés } \\
\text { sont impliquées } \\
\text { dans la gouvernance }\end{array}$ & $\begin{array}{l}\text { Quasi-absence de gouvernance } \\
\text { collaborative }\end{array}$ & $\begin{array}{l}\text { Gouvernance collaborative } \\
\text { limitée } \\
\text { - Les parents peuvent } \\
\text { participer à la supervision } \\
\text { en tant que sources } \\
\text { d'information }\end{array}$ \\
\hline $\begin{array}{l}\text { Organisation fondée sur } \\
\text { les mécanismes du marché } \\
\text { - Droit des usagers à choisir } \\
\text { librement } \\
\text {... mais l'établissement } \\
\text { peut refuser une candidature }\end{array}$ & $\begin{array}{l}\text { Organisation fondée sur } \\
\text { les mécanismes du marché } \\
\text { - Droit des usagers à choisir } \\
\text { librement }\end{array}$ & $\begin{array}{l}\text { Organisation fondée sur } \\
\text { les mécanismes du marché } \\
\text { - Droit des usagers à choisir } \\
\text { librement }\end{array}$ \\
\hline
\end{tabular}




\section{APPROCHES POLITIQUES ET TRADITIONS HISTORIQUES : TENTATIVES D'EXPLICATION}

Dans les trois pays, la citoyenneté active, la participation des usagers et l'adaptation à leurs besoins sont des objectifs centraux visés par l'État pour ses services publics. Pour atteindre ces objectifs, ces États mettent en place des politiques visant à organiser et gérer les services publics de façon différente du modèle traditionnel, dans lequel le secteur public à la fois finance et fournit les services. Les autorités nationales au Danemark, en Norvège et en Suède réglementent de façon plus ou moins poussée la scolarisation en établissements privés au primaire ou dans le secondaire inférieur (qu'ils soient à but lucratif ou non).

L'impression générale est celle d'une grande similarité d'approche s'agissant des politiques publiques en matière d'éducation privée dans ces trois pays. Le secteur de l'enseignement, en outre, est en partie caractérisé par une organisation fondée sur les mécanismes du marché, puisque les usagers ont le droit de choisir leur établissement - même si ce choix est limité aux établissements homologués par l'État. Ce " marché » des écoles privées subsidiées est donc un quasimarché, qui n'est qu'en partie déconnecté du contrôle étatique.

En dépit de ces similarités, les cadres nationaux qui définissent l'interaction entre le financeur public et les fournisseurs de services privés varient de façon significative, en particulier s'agissant de la liberté de fonctionnement dont jouissent les acteurs privés. Dans quelle mesure sont-ils libres de définir les valeurs et objectifs de leur projet éducatif ? Si ces derniers sont encadrés par des standards nationaux étroits, il n'est guère probable que l'offre éducative soit très diversifiée. Dans de telles conditions, il peut également être difficile de garantir un des éléments centraux d'une citoyenneté active, à savoir la possibilité de choisir au sein d'une offre éducative étendue. Ce dilemme peut également être relié à la question de la fragmentation. Une grande latitude accordée aux établissements privés peut entraîner des phénomènes de fragmentation en termes d'objectifs et de résultats, de pratiques d'éducation et d'instruction, et de formes scolaires, ce qui va dans le sens du souhait d'une diversité de l'offre éducative. Toutefois, les bénéfices de ces types de fragmentation pour les usagers (élèves et parents) dépendent des possibilités qui leur sont données de participer activement et de choisir de façon éclairée. D’un autre côté, ces phénomènes de fragmentation peuvent être considérés comme une menace contre les principes égalitaristes qui défendent l'idée d'un système scolaire unifié et d'un droit universel à recevoir la même éducation, indépendamment des ressources financières et du lieu de résidence. Ces deux idées d'égalité et d'universalité ont, historiquement, été très ancrées dans le modèle scandinave d'éducation, et ont servi d'arguments puissants en faveur de strictes régulations publiques. 
Il existe donc un certain nombre de différences entre les pays scandinaves, s'agissant du degré et du type de fragmentation engendrés par la régulation nationale des opérateurs scolaires privés. Il nous reste à examiner les raisons qui expliquent ces différences nationales. Certaines de ces raisons sous-jacentes sont probablement d'ordre politique . $^{3}$.

On peut en effet se demander si la composition des gouvernements nationaux et la représentation des différents partis en leur sein ont affecté les politiques éducatives. Cette question est particulièrement importante si l'on considère les changements qui ont affecté la Suède. Ce pays est en effet peut-être la meilleure illustration de l'impact des choix politiques sur l'évolution de l'État providence. Pendant les années 1990, "la liberté de choisir » et « la concurrence » étaient les slogans dominants dans le paysage politique suédois. Ils ont conduit à diverses réformes, y compris dans le domaine éducatif, conduites par le gouvernement Bildt de centre-droit (1991-1994), puis, plus tard, par le gouvernement Reinfeldt, également de centre-droit (2006-2014). En conséquence, la Suède est passée d'un modèle d'État providence, dans lequel le secteur public fournissait la quasi-totalité des services, à une société de services, dans laquelle le rôle des organismes privés à but lucratif a augmenté et où les usagers eux-mêmes se sont vu accorder la possibilité d'agir sur l'offre de services à travers leurs choix de fournisseurs.

L'impact des changements de gouvernement peut également être observé en Norvège : le deuxième gouvernement de centre-droit Bondevik (2001-2005) a facilité l'établissement d'écoles privées qui n'étaient ni confessionnelles ni fondées sur une pédagogie alternative. Le deuxième gouvernement de centregauche Stoltenberg (2005-2013) est revenu sur cette décision, avant que le gouvernement de droite actuel Solberg (depuis 2013) ne revienne à une politique à nouveau plus libérale.

Si la politique partisane joue un rôle, elle ne peut toutefois expliquer à elle seule les différences de politiques éducatives entre les pays scandinaves. Ainsi, le gouvernement Solberg, en Norvège, n'a pas autorisé les établissements privés à faire du profit à partir de financements publics et a maintenu les critères très stricts d'homologation. Durant la campagne électorale de 2013 en Norvège, tous les partis ont décrit la situation suédoise comme indésirable. La gauche norvégienne a accusé la droite de vouloir introduire des réformes sur le modèle suédois, tandis que la droite mettait en avant ses différences avec les partis de droite en Suède. Cette hypothèse que les changements d'orientation politique ne sont pas uniquement dus à des changements de gouvernement se vérifie aussi en Suède. Les réformes libérales qui ont conduit au développement du secteur des établissements à but lucratif ont en effet été, dans une large mesure, acceptées par les sociaux-démocrates (Wiborg, 2013).

3. Sur ce sujet, voir Segaard \& Saglie 2017. 
Le cas danois illustre bien l'importance finalement limitée de la politique partisane. Depuis 2001, ce sont principalement des gouvernements de droite qui se sont succédé (2001-2011 et depuis 2015). Toutefois, ils n'ont pas libéralisé le marché de l'éducation de manière comparable à ce qu'a faut la Suède ${ }^{4}$. Le cas danois met donc en lumière l'importance des traditions historiques nationales. Le pays a une longue tradition d'écoles privées à but non lucratif, qui coexistent avec des pensionnats (privés non lucratifs); et cette histoire particulière influence le discours politique actuel. Cette tradition date du milieu du XIX ${ }^{\mathrm{e}}$ siècle et reflète la philosophie de N.F.S. Grundtvig et de Christen Mikkelsen Kold. Aujourd'hui, il existe au Danemark une grande variété idéologique des établissements à but non lucratif - depuis les mouvements alternatifs de gauche jusqu'aux chrétiens ou aux libéraux. Chacun de ces groupes dispose d'alliés politiques, ce qui conduit à un consensus trans-partisan sur l'importance du secteur privé à but non lucratif. Même si ce secteur est nettement moins développé en Norvège, des éléments similaires peuvent être observés dans ce pays. Dans le cas norvégien, la position centrale du parti populaire chrétien et du parti libéral semble jouer un rôle important. Ces deux partis se sont traditionnellement montrés sceptiques face aux tentatives de libéraliser le marché de l'éducation, et leur priorité a toujours été de protéger les institutions à but non lucratif des effets de cette marchandisation. À l'inverse, en Suède, de tels freins au développement d'alternatives à but commercial semblent absents.

Pour résumer, les histoires individuelles de chaque pays semblent conditionner leurs trajectoires et limiter les options discutées ci-dessus. Cette observation est en accord avec la littérature relative aux différents modèles d'État-providence, dont l'un des arguments principaux est que « l'histoire et la politique expliquent tous deux ${ }^{5}$ ces modèles (Arts et Gelissen, 2010).

\section{BIBLIOGRAPHIE}

ARTS Wil A., GELISSEN J. (2010) : "Models of the welfare state ", in Castles, Leibfried, Lewis, Obinger et Pierson (eds.), The Oxford Handbook of the Welfare State, Oxford : Oxford University Press.

BALLE T. (2012) : Notat om folkeskolerne i Danmark, Finland, Norge og Sverige, paper, Aarhus University, Denmark.

SAHLGREN Gabriel H. (2016) : Regulation and funding of independent schools. Lessons from Sweden, Vancouver : Fraser Institute.

4. Cependant, au cours de la dernière décennie, des politiques publiques visant à promouvoir la concurrence entre les écoles du secteur public ont été mises en œuvre au Danemark. C'est un facteur contextuel crucial pour comprendre l'état actuel de l'offre privée d'éducation dans ce pays, même si ces initiatives politiques ne concernent pas directement le secteur des écoles privées. Néanmoins, la promotion de la concurrence dans le secteur public n'a pas été couplée à une réforme majeure de la libéralisation du secteur des écoles privées, comme on le voit en Suède.

5. Traduction de " history and politics matter ». 
SEGAARD S. B., SAGLIE J. (2017) : « Education and elderly care in Denmark, Norway and Sweden: National policies and legal frameworks for private providers ", in Sivesind et Saglie (eds.), Promoting active citizenship? Markets and choice in Scandinavian welfare, London : Palgrave Macmillan UK.

SEGAARD S. B. (2015) : Skole og eldreomsorg i Skandinavia. Nasjonale føringer for ikke-offentlige aktører, report 2015:07, Oslo : Institute for Social Research.

SØRENSEN E., TORFING J. (2008) : «Introduction: Governance Network Research: Towards a Second Generation », in Sørensen et Torfing (eds.), Theories of Democratic Network Governance, Hampshire/New York : Palgrave Macmillan.

THØGERSEN M. (2017) : "Primary schools in Scandinavia: Local governing and different types of providers ", in Sivesind et Saglie (eds.), Promoting active citizenship? Markets and choice in Scandinavian welfare, London: Palgrave Macmillan UK.

UNESCO (2010) : Citizenship Education for the 21st Century.

VOLCKMAR N., WIBORG S. (2014) : «A Social Democratic Response to Market-Led Education Policies: Concession or Rejection? ", in Blossing, Imsen et Moos (eds.) : The Nordic Education Model: "A School for All" Encounters Neo-Liberal Policy, Springer Science+Business Media Dordrecht.

WIBORG S. (2013) : "Neo-liberalism and universal state education: the cases of Denmark, Norway and Sweden 1980-2011 », Comparative Education 49(4):407-423. 
